\title{
Nasal and Buccal Drug Delivery: Management Forum Conference
}

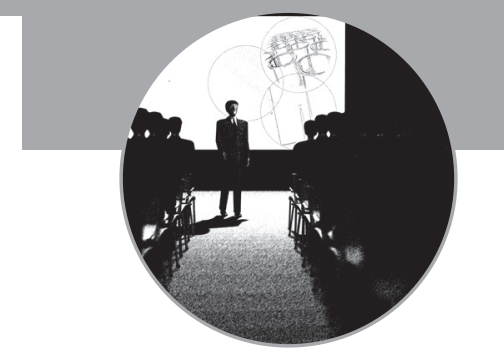

\author{
Nasal and Buccal Drug Delivery: Management Forum Conference \\ Royal Automobile Club, London, UK 23 \& 24 April 2012
}

The scope of the conference (Nasal and Buccal Drug Delivery Conference, Management Forum; Chairs Franz Merkus and Julie Suman) was to consider innovations in drug delivery via the nose and oral cavity, notably for the delivery of vaccines, antimalarials and rapidly acting sedatives. Presentations from experts from academia, government agencies and commercial organisations were made over the 2 days. The advantages of both routes were ease of application, patient acceptability and no requirement to produce sterile products. These routes worked best for drugs that are water soluble - but with some lipophilicity - only require low doses, are acceptable to the patient and have low irritancy (particulary for the nasal route). Challenges relate to the effectiveness of deposition from the delivery systems and the efficient clearance mechanisms. It was concluded that for many drugs, buccal and nasal delivery could become the route of choice for their application; vaccines, in particular, appear to show promise for nasal delivery.

Frans Merkus (Leiden Centre for Drug Research, Leiden, The Netherlands) introduced the conference organised by Management Forum Ltd, the scope of which was to consider innovations in drug delivery via the nose and oral cavity. A particular focus would be recent advances in the area, notably in delivering vaccines, antimalarials and rapidly acting sedatives.

The conference opened with a contribution from Daniel Wermeling (University of Kentucky, KY, USA) who provided the background to nasal drug delivery. In particular, he identified the limitations of the route, such as how it can only be used for drugs that are potent ( $<20 \mathrm{mg}$ ), have a molecular weight of $<1000 \mathrm{Da}$ and good water and lipid solubility. He also discussed the variation in the shape of the nasal cavity between individuals making it difficult to control air flow and, hence, deposition. This route for the emergency delivery of naloxone, an opiate antagonist, was highlighted. The suitability of in vivo models, notably the laboratory rat, were considered and significant differences in absorption profiles between these and human subjects noted.

John Smart (University of Brighton, Brighton, UK) discussed the issues around oromucosal drug delivery. He considered the need for using formulations that were acceptable to the patients (e.g., taste and mouth-feel), the relative impermeability of oral mucosa relative to the rest of the GI tract and the role of salivary washout in clearing drugs. However, oromucosal drug delivery is good for potent drugs with the correct physicochemical properties (similar to those required for nasal absorption), or when a local effect is needed and bioadhesive formulations were identified as a good way to enhance retention and therapy.

Vitaliy Khutoryanskiy (University of Reading, Reading, UK) discussed the development of longer acting bioadhesive formulations using polymer blends, notably polyacrylic acids with non-ionic water-soluble polymers that can produce intermacromolecular complexes, which can regulate bioadhesive performance. He also described a range of tensiometer and simulated in-use systems that can be used to assess bioadhesive performance, including the use of hydrogel mimics of mucosal surfaces.

Chris Collins (Q Scientific Consulting Ltd, Harrogate, UK) discussed nasal and buccal animal models for in vivo testing, with regard to the non-clinical assessment of formulations. He identified issues around the real differences in nasal cavities between standard animal models and humans, in terms of both anatomy and size, which meant that extrapolation from one to another must be undertaken with caution. There are also differences in the oral cavity, for example in size and the nature of the mucosa. Thus, care must be taken in both distribution and toxicological evaluations of formulations in such models.

Els Adriaens (University of Ghent, Ghent, Belgium) discussed the use of models to test irritation and toxicity of buccal and nasal formulations. Adriaens noted the drive to reduce the

\section{John D Smart}

School of Pharmacy \& Biomolecular Sciences, Lewes Road, Brighton, BN2 4GJ, UK

*Author for correspondence:

Tel.: +4401273642091

E-mail: john.smart@brighton.ac.uk 
use of animals in experimental studies, replacing them with validated in vitro models and, in particular, she discussed the idea of using a slug model (Arion lusitanicus) for such evaluations. The slug outer wall is a mucus-secreting ciliated columnar epithelium similar to that of the lining of much of the respiratory tract, including that found in parts of the nasal cavity. Exposure of slugs to various agents in solution was used to evaluate toxicity, in terms of increased mucus secretion and the production of certain biochemical markers of irritancy (lactate dehydrogenase and alkaline phosphatase). There appeared to be a reasonable correlation between the irritancy found in human volunteers and that found in this model. The discussion considered the ease of culturing such slugs in the laboratory and establishing the real comparability of slug walls and respiratory mucosa.

Daryl Bendel (Xidea Solutions, Bushey, UK), discussed the development of an artemether sublingual spray to treat malaria in the developing world. He identified the importance of this in terms of the number of children who die in subSaharan Africa each year $(655,000$ in 2010). The drug, artemether, was one of several extracted from the Chinese medical herb qinghaosu - used to treat malaria in the acute phase and can save lives if administered in time. The sublingual spray, containing the drug along with a medium chain triglyceride and a flavouring levomenthol, was seen as a means by which treatment could be provided quickly away from the hospital environment, without the need for expensive or relatively complex parenteral treatment. In the discussion, the likelihood of the administered drug being swallowed and absorbed in the lower GI tract rather than sublingually was considered, as was the affordability of such a system in poorer countries.

Ronald Van der Geest (3D-PharmXchange, Tilburg, The Netherlands) discussed how pharmacokinetics (drug concentration vs time) and pharmacodynamics (drug concentration vs effect) could be used to produce models to predict dose and dosing intervals, using midazolam as a model.

Julie Suman (Next Breath LLC, MD, USA) covered nasal deposition studies in vitro. She discussed the use of nasal casts, in vitro models of the nasal cavity, which could be used to assess deposition from nasal sprays. In her laboratories she has coated these with ethanol/glycerol, to simulate the mucus coating. Imaging techniques, CAT/MRI scans or gamma scintigraphy can be used to assess deposition in vivo, and the data from this used to confirm nasal cast studies. The need to characterize the nasal spray was noted in terms of the drug mass per actuation, the droplet/particle size, the spray pattern and the plume geometry. She noted how the requirements to understand nasal and buccal deposition by regulatory bodies were becoming more stringent, in particular, avoiding deposition in the lungs on nasal administration.

Rene Bommer (PharmAccel Consulting, Moos, Germany) discussed the various nasal and buccal aerosol drug-delivery systems available. He began by pointing out the growing importance of the transmucosal route for drug delivery, which, in 2014, was predicted to be worth over US $\$ 40$ million, while the global sale of nasal drug formulations was worth $\$ 8.5$ billion globally in 2010. He felt that the industry would move towards the use of the nasal cavity as an alternative route for existing drugs as well as device technology development. He particularly identified the move to preservative-free devices that prevented the ingress of microorganisms and the means by which they could be tested. $\mathrm{He}$ noted that the formulation needs to be primed to get a full dose out at the beginning of therapy and that the doses can also be reduced as the device approaches empty. The majority of the systems that required patients to mechanically activate them (e.g., where patients have to squeeze or blow into them to expel the active material) were susceptible to variation, which could alter their distribution. This would be less of an issue where an active 'propellant' system was incorporated.

Tim Sullivan (Mystic Pharmaceuticals, TX, USA) developed an aerosol device (VeriDoser ${ }^{\mathrm{TM}}$ and $\mathrm{VR}^{2} 2^{\mathrm{TM}}$ ) that could be used for a variety of applications, including nasal distribution. $\mathrm{He}$ said that the aim of his company was to develop user-friendly devices that were easy to use and aided patient compliance. The formulation he described consisted of a blister pack containing a 'Vjet' that allowed distribution of fine sprays of solids or liquids, in a relatively lightly engineered device.

Regina Scherlieb (Christian Albrecht University, Kiel, Germany) described the development of dry-powder formulations for nasal vaccinations. These were seen as a good alternative to injections, as they are non-invasive, there was no need for sterilization and there was evidence of a good immune response via the nasal route. There was also evidence of nanoparticle absorption; at size ranges approximately $<200 \mathrm{~nm}$ that were treated like viruses, while if 
slightly larger $(>500 \mathrm{~nm})$ were treated like bacteria and, thus, both cellular and humoral immune responses could be produced. Absorption of larger particles $<10 \mu \mathrm{m}$ could occur via transcytosis by M cells. Scherlieb described a system that consisted of a model antigen (bovine or egg albumin) along with chitosan as an adjuvant forming the nanoparticle, combined with a carrier sugar of a size that ensured disposition in the nasal passages. Absorption of these particles was via a specific route associated with the 'nasal associated lymphoidal tissue'. When incorporated into an appropriate delivery device, good deposition was found in vitro in a nasal cast and is now being assessed in vivo in an animal model.

Rene Van der Linden (Medir, Doorn, The Netherlands) described an intranasal midazolam formulation that involved producing higher concentrations of aqueous solutions that could be aerosolized and used to produce sedation or treatment of acute epilepsy. The nasal version was much more convenient for the patient. This was found to be comparable to injectable versions of midazolam, generating lower peak plasma levels, although remaining effective, without producing a second peak in the plasma concentration-time curve indicative of gastrointestinal absorption of a swallowed component. This, he concluded, was due to the low volume of the administered solution.

Degenhard Marx (Aptar Pharma, Radolfzell, Germany) discussed the various intranasal devices available for vaccine delivery. There is a strong case for developing nasal vaccines as opposed to normal injectables as they used less material, were easier to store (dry powders may not need refrigeration), were much easier to administer, did not need to be sterilized and were as effective and easier to dispose of. He described that for good nasal vaccination, a liquid should be applied that was $<100-\mu l$ in volume, needed a spray with good nasal deposition, should be administered into both nostrils, must have packing that promotes stability and the spray must be stable and deposited mainly in the nose with little reaching the lungs. To this end, he described work that suggested that the angle of administration and the plume angle could affect deposition efficiency. He described the simplest system, effectively a standard syringe with an adaptor in place of the needle to facilitate production of the spray, which could be used to deliver injectable liquids. The disadvantage of these being that administration and deposition is less precise, there is a large 'dead space' and they cannot be used for solids, which has led to the development of more advanced systems such as the unit-dose system (UDS) blister, bi-dose system, UDS liquid and a possible multidose vaccine device that could be attached to a standard vaccine vial. $\mathrm{He}$ considered the advantages of using dry-powder devices, similar in concept to those used for inhalation therapy, which would enhance stability and has the potential to allow more precise deposition. He described the formulations available for dry-powder vaccine administration, such as the UDS powder. He concluded by saying that the industry was focussed on injectable vaccines, but really there was a good market opportunity for developing nasal vaccines as a much more acceptable, cheaper, easier-to-use and a practical alternative.

Rudiger Nave (Nycomed, Konstanz, Germany) described how the corticosteroid drug ciclesonide, used to treat asthma in the lungs and allergic rhinitis in the nose was actually a prodrug and needed to be converted by an esterase to its active metabolite, desisobutyryl ciclesonide, to have an effect, but then could be deactivated by fatty-acid conjugation. He showed how this was tested to demonstrate this conversion in various nasal models and administration of a hypertonic solution seemed to enhance the effect of this drug.

Susan Cooper (Huntingdon Life Sciences, Huntingdon, UK) described the practicalities of in vivo nasal and buccal safety evaluation. She pointed out that the vast majority of animal research was completed on rodents, with physiologically more relevant animals being much less popular, which meant that differences in behavior between the animal and human models (discussed in previous presentations) could be an issue. She described the feasibility and problems associated with toxicity and distribution studies in such animal models and procedures that could be used to undertake these.

From the presentations it is apparent that there are many opportunities to systemically deliver a number of drugs with the required properties via both routes. Typically these properties are:

- Small (<1000 Da);

- Having both hydrophilic and lipophilic properties (to dissolve and then cross the buccal or nasal mucosae);

- Sufficient potency to be effective in relatively small doses, due to the size of delivered dose (particularly nasal) and the nature of the barrier (particularly buccal); 
- Acceptable to the patient, in terms of taste (including nasally if excess solution runs into the mouth) and odor;

- Low toxicity, in particular the nasal route would not be suitable for irritant materials.

The advantage of both routes are ease of application, patient acceptability (avoiding injections) and no requirement to produce sterile products. In addition to the drug requirements, challenges relate to the effectiveness of the delivery systems and the efficient clearance mechanisms that exist in both areas (typically, clearance time is $15 \mathrm{~min}$ in the nasal cavity).
For a range of drugs, buccal and nasal delivery could become the route of choice for their application and vaccines in particular appear to show promise for delivery via the nasal route.

\section{Financial \& competing interests disclosure}

The author has no relevant affiliations or financial involvement with any organization or entity with a financial interest in or financial conflict with the subject matter or materials discussed in the manuscript. This includes employment, consultancies, honoraria, stock ownership or options, expert testimony, grants or patents received or pending, or royalties. No writing assistance was utilized in the production of this manuscript. 